\title{
Sex Differences in Whole Body Gait Kinematics at Preferred Speeds
}

\author{
Dustin A. Bruening \\ Brigham Young University - Provo, dabruening@byu.edu \\ R. Frimenko \\ C. Goodyear
}

A. Fullenkamp

Follow this and additional works at: https://scholarsarchive.byu.edu/facpub

Part of the Exercise Science Commons

\section{Original Publication Citation}

Bruening DA, Frimenko RE, Goodyear CD, Bowden DR, Fullenkamp AM. Sex differences in whole body gait kinematics at preferred speeds. Gait Posture. 2015 Feb;41(2):540-5. doi: 10.1016/ j.gaitpost.2014.12.011. Epub 2014 Dec 17.

\section{BYU ScholarsArchive Citation}

Bruening, Dustin A.; Frimenko, R.; Goodyear, C.; and Fullenkamp, A., "Sex Differences in Whole Body Gait Kinematics at Preferred Speeds" (2015). Faculty Publications. 1622.

https://scholarsarchive.byu.edu/facpub/1622

This Peer-Reviewed Article is brought to you for free and open access by BYU ScholarsArchive. It has been accepted for inclusion in Faculty Publications by an authorized administrator of BYU ScholarsArchive. For more information, please contact ellen_amatangelo@byu.edu. 


\title{
Sex differences in whole body gait kinematics at preferred speeds
}

\author{
Dustin A. Bruening, Rebecca E. Frimenko, Chuck D. Goodyear, David R. Bowden, Adam M.
} Fullenkamp

\begin{abstract}
Studies on human perception have identified pelvis and torso motion as key discriminators between male and female gaits. However, while most observers would advocate that men and women walk differently, consistent findings and explanations of sex differences in gait kinematics across modern empirical studies are rare. In the present study we evaluated sex differences in whole body gait kinematics from a large sample of subjects (55 men, 36 women) walking at self selected speeds. We analyzed the data through comparisons of discrete metrics and whole curve analyses. Results showed that in the frontal plane, women walked with greater pelvic obliquity than men, but exhibited a more stable torso and head. Women had greater transverse plane pelvis and torso rotation as well as greater arm swing. Additional sex differences were noted at the hip and ankle. These kinematic results are in line with anectdotal observations and qualitative studies. In order to understand these observations and substantiate some of the explanations previously set forth in the biomechanics literature, we also explored possible reasons for dynamic sex effects, and suggested applications that may benefit from their consideration.
\end{abstract}

Keywords: Gender recognition; Gender differences; Torso sway; Pelvic obliquity; Locomotion

\section{Introduction}

There is a widely held perception that men and women walk differently. Evidence supporting this assertion began with studies by Murray et al. [1] and [2] in the 1960s. Most notably, these studies suggested that males exhibit greater torso sway and females greater pelvic obliquity range of motion (ROM). In the 1970s, several human perception studies (e.g. [3]) showed that untrained observers were able to correctly identify subject sex from limited dynamic gait 
information without contextual cues such as clothing or hairstyle. Seeking to determine a summary variable(s) that explained observers' innate abilities to recognize sex differences, Mather and Murdoch [4] and Troje [5] both concluded that, in line with Murray's early assessment, frontal plane pelvis and torso motions were the most critical discriminators between male and female gaits.

More recently, however, empirical studies on gait kinematics have found few consistent sex differences, and have not fully supported the above historical observations. Most striking, three studies have specifically evaluated torso sway sex differences [6], [7] and [8], none finding increased ROM in males. Individual study findings of sex differences in other joints, such as sagittal plane hip [9] and knee [10] and [11] ROM have also not been substantiated by additional studies (e.g. [7], [10], [12] and [13] and [9], [12], [13] and [14], respectively). Arguably the only metric showing some consistency across studies is that of pelvic obliquity, where four [13], [15], [16] and [17] out of seven [7], [18] and [19] studies found increased female ROM. The reasons behind this likely sex difference are also not completely clear, with hypotheses ranging from evolutionary [5] and socio-cultural factors [1] to walking efficiency [17], [20] and [21]. Among other investigated joints, only sagittal plane ankle [9], [10], [22] and [23] and transverse plane hip excursions during early stance [13], [22] and [24] appear to show, across multiple studies, the potential for true sex effects at preferred walking speeds. The possibility of sex differences in other upper body measures, such as arm swing [20], have not been evaluated. Thus, while the lay observer would almost certainly advocate that sex differences exist in walking kinematics, there is still a need to quantify these differences, confirm early historical and anecdotal observations, and seek further clarification of the underlying reasons for these sex differences.

Gait differences between sexes are of interest in a variety of clinical applications. In instrumented gait studies, male and female subjects are often pooled for analysis or normative comparisons, yet there may be specific instances that warrant separation. Sex differences exist in many pathology incidence rates such as osteoarthritis [25], ACL tears [26], and low back pain [27]. Some of these sex differences may be manifest in gait (e.g. [24]) and thus influence treatment and rehabilitation strategies. Similarly, the design of sex-specific joint replacements, prosthetics, and robotic exoskeletons for walking rehabilitation might also be guided by movement differences. Beyond clinical applications, other industries have a vested interest in kinematic sex differences, including psychology [3], [4] and [28], surveillance and tracking [29], targeted advertising [30], and animation [31].

In order for sex differences to be utilized effectively in these applications, they need to be consistently identified and understood. The purpose of the present study, therefore, was to compare whole body kinematic measures between adult men and women walking at preferred speeds. We sought to evaluate specific historical qualitative and anecdotal observations, fill in gaps and provide additional consistency to the existing empirical body of literature, and further explore underlying reasons for possible sex differences. We hypothesized that women would have substantially increased pelvic obliquity, reduced torso sway, and increased arm swing compared to men. 


\section{Methods}

\section{Subjects and protocol}

One hundred healthy subjects were recruited around Wright Patterson Air Force Base.

Exclusionary criteria included the presence of orthopedic injuries or any medical condition that could affect typical gait. Seven subjects were excluded post-hoc due to either visible medical conditions that were not reported (5), or extreme outliers for walking speed (2) and BMI (2), leaving a total of 91 subjects for analysis (36 F, $55 \mathrm{M}$, Table 1). Just over half of the subjects were members of the U.S. military. All subjects were volunteers and signed Institutional Review Board approved consent forms.

Table 1. Demographics.

$\begin{array}{lll} & \text { Female } & \text { Male } \\ \text { N } & 36 & 55 \\ \text { N military } & 18(50 \%) & 34(62 \%) \\ \text { N caucasian } & 26(72 \%) & 45(82 \%) \\ \text { Age }(\mathrm{yr}) & 28.4 \pm 8.3 & 30.7 \pm 10.6 \\ \text { Height }(\mathrm{m}) & 1.67 \pm 0.07 & 1.81 \pm 0.07 \\ \text { Weight }(\mathrm{kg}) & 65.1 \pm 11.5 & 84.1 \pm 12.2 \\ \text { BMI }\left(\mathrm{kg} / \mathrm{m}^{2}\right) & 23.2 \pm 3.5 & 25.7 \pm 3.3\end{array}$

A total of 68 retro-reflective markers were affixed to each subject according to a modified Helen Hayes type (i.e. sparse) marker set. For the subject's extremities, markers were placed on the medial and lateral aspects of each joint with an additional marker on the mid segment for tracking, consistent with typical descriptions of sparse marker set configurations (i.e. derived from [19] and [32]). For the subject's midsection, markers were placed over the ASIS and sacrum (pelvis), manubrium, xyphoid, C7, and T8 (torso), with three markers on the head.

All subjects wore comfortable walking shoes. After a static pose and a short, accustomizing warm up, each subject was asked to walk at a preferred, or comfortable (i.e. self-selected) speed across a $15 \mathrm{~m}$ walkway. Four walking trials were performed. Marker trajectories were captured at $120 \mathrm{~Hz}$. using 18 Motion Analysis Raptor-E cameras (Motion Analysis Corp, Santa Rosa, CA).

\section{Data analysis}

Data processing was performed in Visual 3D software (C-Motion, inc. Germantown MD). Static trial marker trajectories were averaged over a small frame range, while walking marker trajectories were filtered using a low pass Butterworth Filter (6 Hz cutoff frequency). A 15segment full body model was created from the static pose. The pelvis, torso, and head reference 
frames were aligned to the laboratory reference frame in the static pose. Shoulder joint centers were calculated using an offset modified from Rab et al. [33], while hip centers were calculated from Harrington et al. [34]. Extremities were aligned with the segment's long axis and medial/lateral joint markers creating the frontal plane. The model was applied to each walking trial, tracking each segment with all of its associated markers. Joint angles were then calculated using an Euler/Cardan angle sequence (1-flex/ext, 2-Ab/ad, 3-Int/Ext). Angle trajectories were broken into gait cycles and time-normalized to percent gait cycle.

ROM was calculated for each angle across the gait cycle, except for hip rotation, which was calculated from initial contact to mid-stance. The mean ROM for each subject (across, on average, 20 cycles per subject) was used in the group analysis. Spatiotemporal variables were calculated and non-dimensionalized using leg length (calculated from static pose: hip to knee plus knee to ankle) according to the scheme proposed by Hof [35]: stride length normalized by leg length, velocity by the square root of $g \times$ leg length, and cadence by the square root of $g / l e g$ length. Root mean square (RMS) accelerations [20] of each midsection segment were calculated by double differentiating the segment center of mass. Vertical center of mass (COM) excursions were calculated and also normalized by leg length.

Two sample t-tests were used to detect sex differences among all spatiotemporal, acceleration, ROM, and COM variables of interest $(\alpha=0.05)$. Spatiotemporal variables included walking speed, stride length, cadence, and step width. RMS accelerations were calculated in the medial/lateral (M/L) and anterior/posterior (A/P) directions for the pelvis, torso, and head segment center's of mass. ROM was evaluated in all three planes for the pelvis, torso, waist (defined as the relative angle between the torso and pelvis), and hip, and in the sagittal plane for the knee, ankle, shoulder, and elbow. Based on results suggesting arm swing asymmetry [36], shoulder and elbow ROM were evaluated using $2 \times 2$ between-within ANOVAs to concurrently assess the effects of both sex and side. Pelvis and upper body joint angles were further evaluated using a whole curve analysis, where mean \pm standard error bands where plotted and $t$-statistics were calculated at each point of the gait cycle. A significance cutoff was set at $t= \pm 2.63$, corresponding to $\alpha=0.01$.

\section{Results}

Men and women walked at nearly the same mean preferred speed, men taking significantly longer strides, and women moving with a higher cadence (Table 2A). When accounting for body size, non-dimensional walking speed and stride length comparisons were not significant, but notable, with potentially greater mean values in women compared to men.

Table 2. Means, standard deviations, and p-values from selected spatiotemporal, range of motion, acceleration, and center of mass (COM) variables. Sex differences were evaluated with two sample t-tests $(\alpha=0.05)$, with the exception of shoulder and elbow 
ROM, which were assessed for sex and side differences via $2 \times 2$ ANOVAs (side differences listed in text). * Denotes significant p-values.

\section{(A) Spatiotemporal variables}

\begin{tabular}{|c|c|c|c|}
\hline & Female (mean \pm SD) & Male (mean \pm SD) & $p$-Value \\
\hline Walking speed (m/s) & $1.35 \pm 0.16$ & $1.34 \pm 0.13$ & 0.608 \\
\hline Stride length (m) & $1.43 \pm 0.11$ & $1.49 \pm 0.09$ & $0.014 *$ \\
\hline Cadence (steps/min) & $113.3 \pm 8.0$ & $108 \pm 6.6$ & $<0.001^{*}$ \\
\hline Step width (m) & $0.094 \pm 0.027$ & $0.089 \pm 0.029$ & 0.438 \\
\hline Walking speed (non-dim) & $0.49 \pm 0.06$ & $0.47 \pm 0.05$ & 0.059 \\
\hline Stride length (non-dim) & $1.86 \pm 0.15$ & $1.80 \pm 0.15$ & 0.067 \\
\hline Cadence (non-dim) & $0.53 \pm 0.04$ & $0.52 \pm 0.03$ & 0.338 \\
\hline
\end{tabular}

(B) Joint angle range of motion (ROM), in degrees

\begin{tabular}{llll}
\hline & \multicolumn{2}{c}{ Female (mean \pm SD) Male (mean \pm SD) $\boldsymbol{p}$-Value } \\
Ankle flex/ext & $34.6 \pm 3.5$ & $32.4 \pm 4.5$ & $0.014^{*}$ \\
Knee flex/ext & $67.2 \pm 3.2$ & $68.1 \pm 4.5$ & 0.261 \\
Hip flex/ext & $45.7 \pm 4.5$ & $44.1 \pm 3.9$ & 0.076 \\
Hip ab/adduction & $16.2 \pm 3.4$ & $14.2 \pm 3.1$ & $0.006^{*}$ \\
Hip rotation (loading) & $5.9 \pm 3.2$ & $3.7 \pm 2.2$ & $<0.001^{*}$ \\
Pelvic tilt & $4.4 \pm 1.3$ & $4.5 \pm 1.2$ & 0.891 \\
Pelvic obliquity & $9.4 \pm 2.6$ & $6.8 \pm 2.1$ & $0.000^{*}$ \\
Pelvic rotation & $14.1 \pm 4.6$ & $12.0 \pm 3.0$ & $0.016^{*}$ \\
Torso flex/ext & $3.6 \pm 0.7$ & $3.3 \pm 0.7$ & 0.104 \\
Torso sway & $3.5 \pm 1.3$ & $4.9 \pm 1.8$ & $<0.001^{*}$ \\
Torso rotation & $7.4 \pm 2$ & $5.8 \pm 2$ & $<0.001^{*}$ \\
Waist flex/ext & $4.4 \pm 0.9$ & $4.1 \pm 1.1$ & 0.241 \\
Waist side bending & $12.4 \pm 2.9$ & $12.1 \pm 2.7$ & 0.641 \\
Waist rotation & $13.5 \pm 5.0$ & $13.7 \pm 3.4$ & 0.784 \\
Shoulder flex/ext & $29.1 \pm 11.3$ & $22.4 \pm 6.9$ & $0.002^{*}$ \\
Elbow flex/ext & $33.6 \pm 10.9$ & $29.7 \pm 8.3$ & 0.056
\end{tabular}

(C) RMS accelerations of torso, pelvis, and head segments, in $\mathrm{m} / \mathrm{s}^{2}$

\begin{tabular}{llll}
\hline & Female (mean \pm SD) & Male (mean \pm SD) & $\boldsymbol{p}$-Value \\
Pelvis M/L & $1.13 \pm 0.30$ & $0.96 \pm 0.22$ & $0.004^{*}$ \\
Torso M/L & $0.60 \pm 0.11$ & $0.62 \pm 0.13$ & 0.495 \\
Head M/L & $0.74 \pm 0.18$ & $0.86 \pm 0.20$ & $0.007^{*}$ \\
Pelvis A/P & $1.75 \pm 0.24$ & $1.61 \pm 0.22$ & $0.004^{*}$
\end{tabular}


(C) RMS accelerations of torso, pelvis, and head segments, in $\mathrm{m} / \mathrm{s}^{2}$

\begin{tabular}{llll}
\hline & Female $($ mean \pm SD) & Male (mean \pm SD) & $p$-Value \\
Torso A/P & $1.43 \pm 0.19$ & $1.28 \pm 0.18$ & $<0.001^{*}$ \\
Head A/P & $0.62 \pm 0.20$ & $0.63 \pm 0.22$ & 0.705
\end{tabular}

(D) Center of mass (COM) excursions

\begin{tabular}{|c|c|c|c|}
\hline & Female (mean \pm SD) & Male (mean & $p$-Value \\
\hline Vertical (cm) & $4.59 \pm 0.79$ & $4.53 \pm 0.67$ & 0.712 \\
\hline Vertical (non-dim) & $5.96 \pm 1.07$ & $5.50 \pm 1.00$ & $0.040 *$ \\
\hline
\end{tabular}

In the lower extremities, women demonstrated significantly greater pelvic obliquity and rotation, hip ab/adduction, hip rotation during loading, and ankle flex/ext (Table 2B). In the upper body, women had significantly less torso sway but more torso rotation. Arm swing was higher in women compared to men, with women having significantly greater shoulder ROM as well as a non-significant, but notable (particularly in light of the whole curve analysis) difference in elbow ROM (Table 2B). Side differences were also evident at the elbow, with left elbow ROM significantly greater than right $\left(33.4 \pm 7.5^{\circ}\right.$ compared with $\left.29.9 \pm 7.3^{\circ}, p<0.001\right)$. No significant side differences were noted at the shoulder $\left(26.1 \pm 6.6^{\circ}\right.$ on the left compared with $25.3 \pm 7.8^{\circ}$ on the right, $p=0.366$ ), and no significant interactions were found between sex and side for either joint $(p>0.127)$.

Women showed a greater $\mathrm{M} / \mathrm{L}$ acceleration attenuation than men from pelvis to head as evidenced by greater RMS accelerations at the pelvis, similar at the torso, and less at the head (Table 2C). Similarly, attenuation differences were apparent in A/P accelerations, with women showing greater pelvis and torso RMS values than men, but similar values at the head.

No significant difference was found in raw vertical COM excursion between sexes, but women had a significantly greater normalized COM excursion than men.

Whole curve plots (Fig. 1) showed sex differences occurring primarily around the peaks, suggesting that the ROM analysis captured the primary differences in most joint angle profiles. Pelvic rotation showed a possible phase shift, with the concave external rotation wave slightly delayed in women compared with men. Torso sway also differed in shape, with women exhibiting an extended period of neutral torso position at midstance, and men transitioning in a more continuous motion. Torso rotation had a similar but reversed trend, with men having a more level internal rotation through midstance. The curve analysis also showed that sex differences in shoulder motion were more pronounced in flexion than extension, while possible elbow differences appeared to occur more in extension. The left/right side differences in elbow ROM were likely due to combined increases in both flexion and extension. 

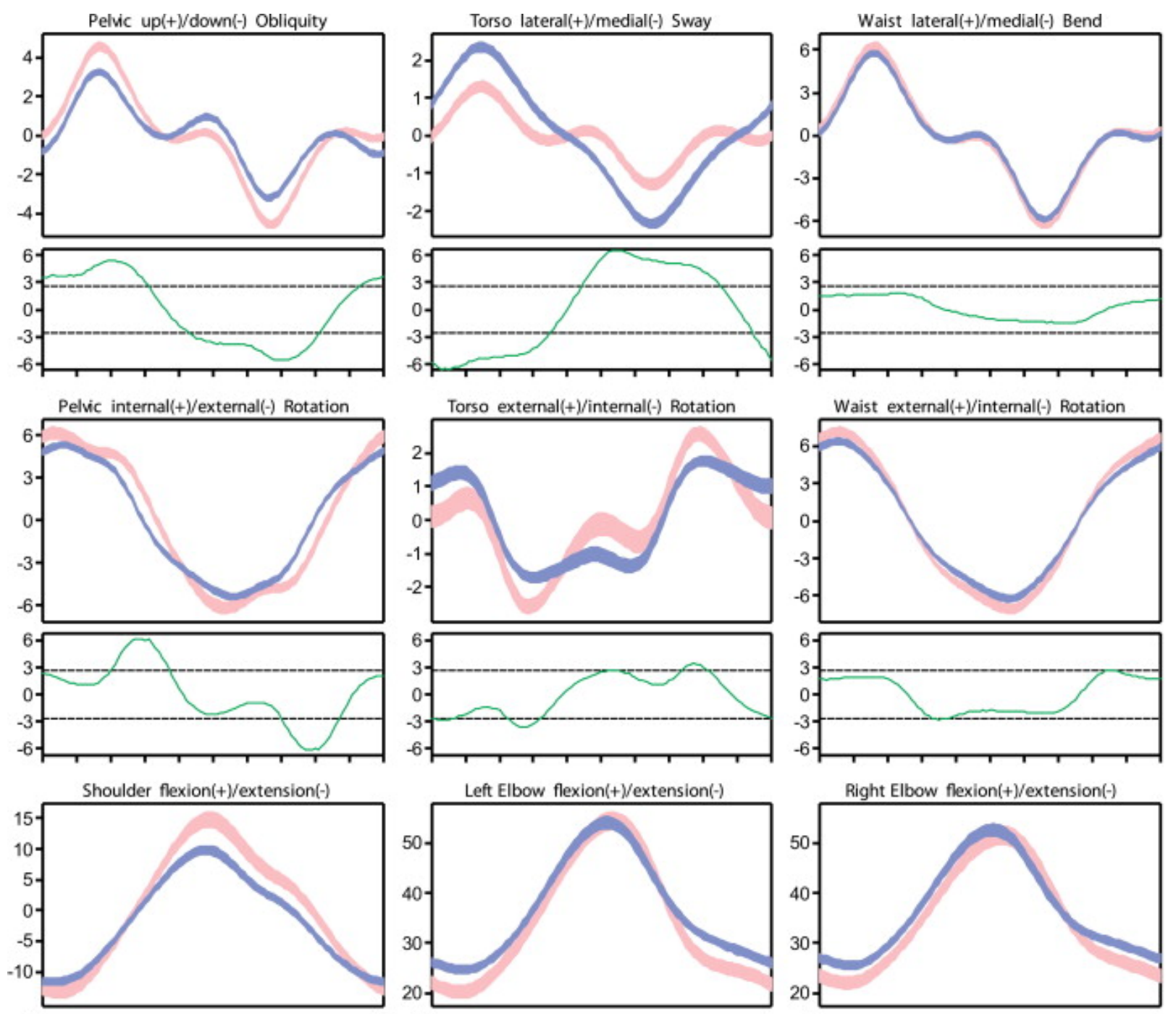

Right Elbow flexion(+)/extension(-)
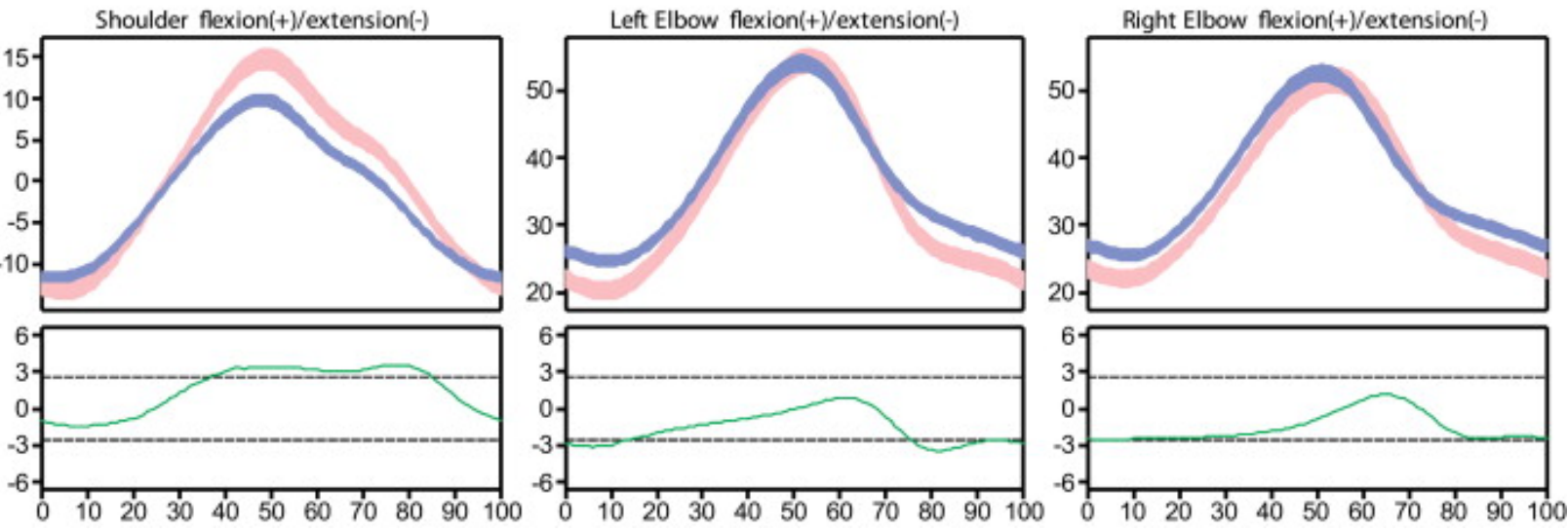

Fig. 1. Mean \pm standard error profiles across the gait cycle for select pelvis and upper body angles (in degrees), allowing for comparison between men (dark blue) and women (light pink). $t$-statistics were calculated at each point in the gait cycle, and are plotted below each curve (green line). A rough significance threshold is shown on the t-statistic graphs at $t= \pm 2.63$, corresponding to a conservative $\alpha=0.01$. Regions on the angle graphs where the t-statistic is above or below this threshold might be considered statistically significant and regions of interest. (For interpretation of the references to color in this figure legend, the reader is referred to the web version of this article.) 


\section{Discussion}

In this study we investigated sex differences in whole body gait kinematics. Our lower extremity results are consistent with several previous studies, further strengthening findings of specific pelvis, hip, and ankle kinematic differences. For upper body measures, however, previous evidence of sex differences comes primarily from qualitative studies and anecdotal observations. In contrast to several recent empirical studies [6], [7] and [8], our results show clear evidence of sex differences in torso and arm motion (Table 2B and Fig. 1) that match historical hypotheses.

Several explanations for sex differences in frontal plane pelvis and torso motion have been posited. Smith et al. [17] hypothesized that greater pelvic obliquity may be a female mechanism to increase metabolic efficiency, as evidenced by a corresponding decrease in vertical COM excursions. However, significant findings in their study were primarily confined to an elderly demographic sample. In our study sample we did not find a sex difference in raw COM excursions, while normalized COM excursions were greater in women than men. The latter effect may be more related to preferred spatiotemporal metrics than to a true COM sex effect, as normalized speed and step length were also slightly greater in women [37]. Mazza et al. [20] and [21] also suggested that women had a possible efficiency advantage, evidenced by more effective acceleration attenuation up the kinematic chain from pelvis to head. Although there were some discrepancies in RMS magnitudes between our results and Mazza's, likely due to measurement location (segment center of mass rather than dorsal surface) and methodology (differentiation of position rather than accelerometry measured), the attenuation patterns were similar. Future studies should determine whether a stable head is also more metabolically efficient. Murray et al. [1] also suggested coupling between frontal plane pelvis and torso motion but for different underlying reasons. They hypothesized that necessary movement of the center of mass from side to side could be accomplished by either moving the pelvis or the torso, the choice of which was likely an "attitudinal”, or socio-cultural characteristic. Psychology literature has also presented some evidence linking pelvis and torso movements to sexual attractiveness [28] and [38], and Troje [5] compared the greater lateral sway in men to evolutionary adaptations of male animals who make themselves appear larger and thus intimidating. Determining whether frontal plane pelvis and torso sex differences are due to aspects of walking efficiency, sociocultural factors, or other possible reasons (e.g. bone structure and muscle mechanics) is difficult, but the amalgamation of findings across fields suggests that interplay among these factors is likely.

The combined effects of pelvis, torso, and waist angles in both the frontal and transverse planes (Fig. 1) suggest that men and women use different control strategies during gait. Interestingly, waist angles were remarkably similar between sexes, suggesting a possible coupled control strategy wherein ideal lumbar or COM kinematics are maintained. This occurred despite sex differences in pelvis and torso kinematics in both the frontal and transverse planes. In the transverse plane, both pelvis and torso rotation showed clear sex differences in ROM as well as in curve shape. While previous literature on frontal plane pelvis kinematics has been fairly consistent in reporting similar results among studies, this has not been the case in the transverse 
plane (Table 3). Only Crosbie et al. [7] reported greater pelvic rotation ROM in women; yet ROM values in both Crosbie et al. and Cho et al. [13] were low in comparison to other normative datasets. Neither Chockalingam et al. [15] nor Smith et al. [17] found significant sex differences. Our whole curve analysis shows an additional phase shift, with women delaying the transition from internal to external rotation as well as the return back to internal rotation. This type of phase shift is not consistent with a faster walking speed or longer step length [39], suggesting a possible true sex effect that should be further evaluated.

Table 3. Comparison of frontal and transverse plane pelvis, torso, and waist ROM findings to previous literature reporting sex differences in overground, self selected speed trials. ${ }^{*}$ Indicates a significant sex difference was reported. ${ }^{A}$ Indicates that ROM was not listed, but approximate values could be gleaned either from reported maximums and minimums, or visually extracted from presented graphs. ${ }^{B}$ Chumanovs study was at a controlled $1.2 \mathrm{~m} / \mathrm{s}$ on a treadmill, rather than overground.

\section{(A) Frontal plane}

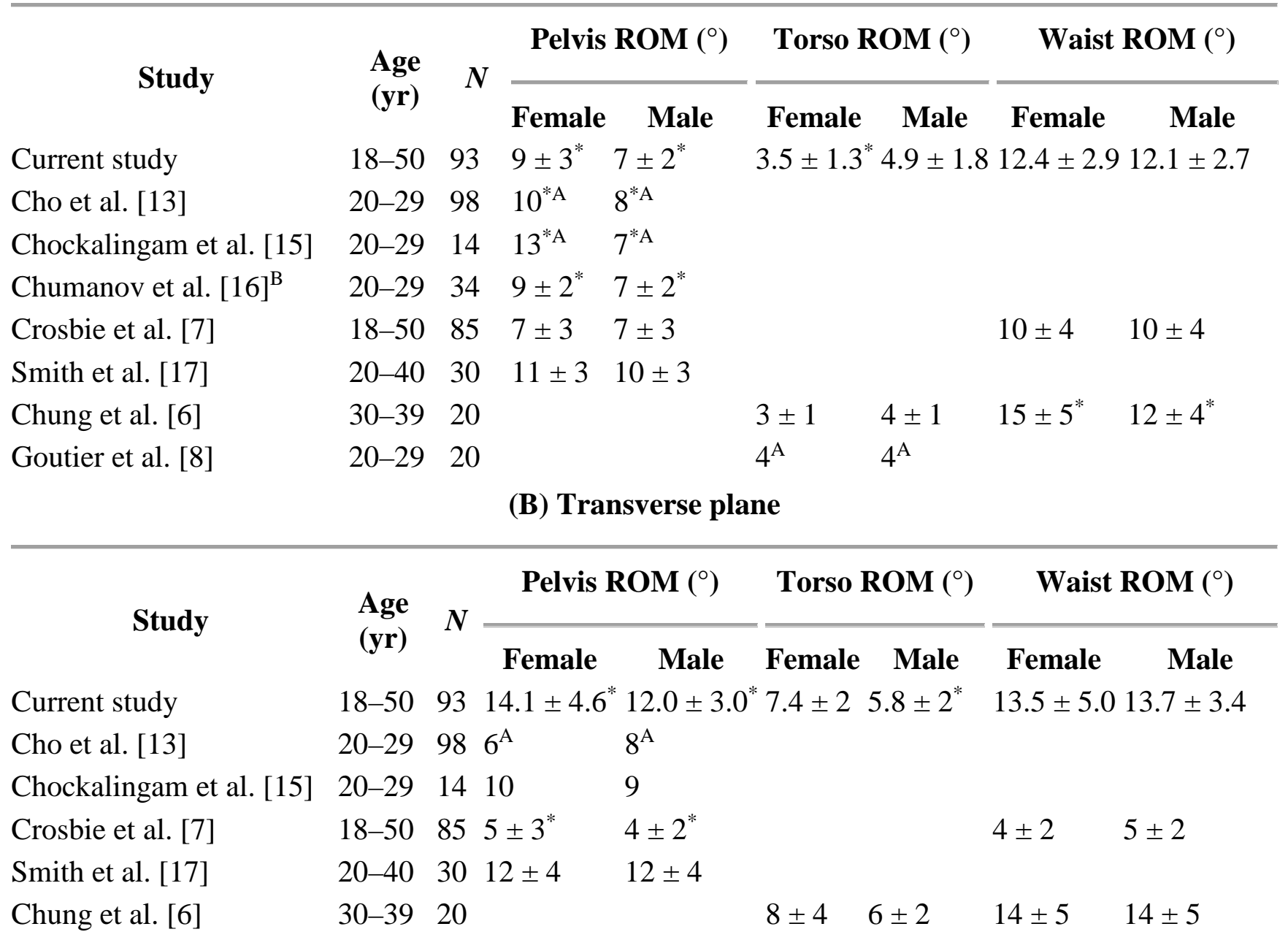


At the hip, female internal rotation was increased from initial contact through loading response. Despite marker set differences (i.e. soft tissue interactions), our findings match those of both Hurd et al. [24] and Roislien et al. [22], further strengthening this finding. The reason for this increase may be structural, related to women's wider pelvis and greater femoral anteversion, or it may be coupled to increased frontal plane motion. Hip ab/adduction ROM was greater in women, although likely primarily driven by the greater pelvis motion. The subtle hip kinematic differences noted here may have implications in injury mechanisms, and may warrant further investigation to determine whether they carry over to running or other athletic movements [24].

Similar to several previous studies [9], [10], [22] and [23], we also found greater ankle ROM in women compared with men. While the difference between sexes was on the order of just $2^{\circ}$ over a $30^{\circ} \mathrm{ROM}$, the presence of this finding across multiple studies is intriguing. In athletics, it is well documented that altered ankle kinematics can affect more proximal joints in the kinematic chain, and be a contributing cause of various knee and hip injuries. It is possible that women, with proportionally shorter feet compared to men [40], require a slightly increased plantarflexion push off angle to maintain effective knee kinematics. The effects of sex differences in both foot structure and ankle motion may merit further investigation, particularly as they relate to injury mechanisms.

Sex differences in arm swing have not been previously evaluated, although Mazza [20] mentions possible anecdotal observations of greater arm ROM in women. Arm swing is likely primarily a passive motion driven by the lower extremities [41]; however, active modulation can result in substantial variations in ROM. Some subjects in our study appeared to suppress arm swing due to awareness of being in a laboratory setting. Still, we found a significant increase in flexion/extension ROM at the shoulder in women, and a p-value of 0.056 at the elbow. It is possible that the higher raw cadence and greater torso rotation noted in women may have simply resulted in greater arm angular velocities and momentum, passively driving arm swing ROM differences. However, the whole curve analysis showed that potential sex differences were primarily confined to peak flexion at the shoulder and peak extension at the elbow ( Fig. 1), suggesting possible structural, muscular, or other sex based influences beyond those expected from passive mechanisms. We also found a right/left side effect, with increased ROM at the left elbow in both sexes, matching a previous finding by Kuhtz-Buschbeck et al. [36]. This finding may also point toward active modulation of arm swing. Future studies utilizing controlled cadence and speed trials, electromyography, and/or forward dynamic simulations may help clarify active vs. passive contributions to arm swing, and help determine whether true sex differences are present.

In this study we focused only on kinematics at self selected walking speeds. The effects of controlled walking speeds or cadences, as well as other factors such as culture or aging, were not evaluated. However, several indicators suggest that the data set collected in this study is representative of the larger United States population. First, the sample is large and fairly diverse demographically (i.e. non-college students). While we did include a large number of military subjects, no substantial clustering or separation was noted in any variables of interest between military and non-military subjects. Second, mean group height, weight, and BMI measures are 
on par with larger national surveys [42]. Finally, preferred pace spatiotemporal measures were also comparable to previous literature. Mean walking speeds were within normal ranges of the pooled means across previous studies [43] as well as similar to several studies used in this discussion for gender comparisons [10], [17] and [20]. Stride length and cadence sex differences are also consistent with previous studies showing similar trends at preferred speeds [10] and [17].

Several clinical applications may benefit from the results of this study. In the frontal plane, separate male and female pelvis and torso normative values may help in some pathology diagnosis and treatment recommendations. For example, a Trendelenburg gait pattern, caused by gluteus medius weakness, is often diagnosed by increased pelvic obliquity ROM. The sensitivity of the diagnosis, and the extent of the weakness, may be better gauged by considering subject sex. Altered frontal plane pelvis and torso kinematics have been noted in subjects with knee osteoarthritis [44], and gait retraining that includes increasing lateral torso sway has been suggested as a compensatory strategy to reduce medial knee loading [45]. The effectiveness of this type of re-training may differ by sex. Lateral sway has also been associated with the risk of falling in the elderly [46]. Research on fall risks as well as pathologies that affect the elderly might also benefit from consideration of sex differences.

\section{Acknowledgments}

Aspects of this effort were funded by the Air Force Research Laboratory under contract number FA86-50-12-D-6354.

\section{References}

[1] M.P. Murray, R.C. Kory, S.B. Sepic .Walking patterns of normal women. Arch Phys Med Rehabil, 51 (1970), pp. 637-650

[2] M.P. Murray, R.C. Kory, B.H. Clarkson, S.B. Sepic. Comparison of free and fast speed walking patterns of normal men. Am J Phys Med, 45 (1966), pp. 8-23

[3] L.T. Kozlowski, J.E. Cutting. Recognizing the sex of a walker from a dynamic point-light display. Percept Psychophys, 21 (1977), pp. 575-580

[4] G. Mather, L. Murdoch. Gender discrimination in biological motion displays based on dynamic cues. Proc. R. Soc. London, Ser. B: Biol. Sci., 258 (1994), pp. 273-279

[5] N.F. Troje. Decomposing biological motion: a framework for analysis and synthesis of human gait patterns. Vis, 2 (2002), pp. 371-387 
[6] C.Y. Chung, M.S. Park, S.H. Lee, S.J. Kong, K.M. Lee. Kinematic aspects of trunk motion and gender effect in normal adults. J Neuroeng Rehabil, 7 (1) (2010), pp. 1-7

[7] J. Crosbie, R. Vachalathiti, R. Smith. Age, gender and speed effects on spinal kinematics during walking. Gait Posture, 5 (1997), pp. 13-20

[8] K.M. Goutier, S.L. Jansen, C.G. Horlings, U.M. Küng, J.H. Allum. The influence of walking speed and gender on trunk sway for the healthy young and older adults. Age Ageing, 39 (2010), pp. 647-650

[9] S.-u. Ko, M.I. Tolea, J.M. Hausdorff, L. Ferrucci. Sex-specific differences in gait patterns of healthy older adults: results from the Baltimore Longitudinal Study of Aging. J Biomech, 44 (2011), pp. 1974-1979

[10] D.C. Kerrigan, M.K. Todd, U. Della Croce. Gender differences in joint biomechanics during walking: normative study in young adults. Am J Phys Med Rehabil, 77 (1998), pp. 2-7 (Association of Academic Physiatrists)

[11] D.B. Kettelkamp, R.J. Johnson, G.L. Smidt, E.Y. Chao, M. Walker. An electrogoniometric study of knee motion in normal gait. J Bone Joint Surg Am, 52 (1970), pp. 775-790

[12] F. Alton, L. Baldey, S. Caplan, M.C. Morrissey. A kinematic comparison of overground and treadmill walking. Clin Biomech (Bristol, Avon), 13 (1998), pp. 434-440

[13] S.H. Cho, J.M. Park, O.Y. Kwon. Gender differences in three dimensional gait analysis data from 98 healthy Korean adults. Clin Biomech (Bristol, Avon), 19 (2004), pp. 145-152

[14] T. Oberg, A. Karsznia, K. Oberg. Joint angle parameters in gait: reference data for normal subjects, 10-79 years of age. J Rehabil Res Dev, 31 (1994), pp. 199-213

[15] N. Chockalingam, F. Chatterley, A.C. Healy, A. Greenhalgh, H.R. Branthwaite. Comparison of pelvic complex kinematics during treadmill and overground walking. Arch Phys Med Rehabil, 93 (2012), pp. 2302-2308

[16] E.S. Chumanov, C. Wall-Scheffler, B.C. Heiderscheit. Gender differences in walking and running on level and inclined surfaces. Clin Biomech (Bristol, Avon), 23 (2008), pp. 1260-1268

[17] L.K. Smith, J.L. Lelas, D.C. Kerrigan. Gender differences in pelvic motions and center of mass displacement during walking: stereotypes quantified. J Women's Health, 11 (2002), pp. 453-458 (gender-based medicine) 
[18] J. Crosbie, R. Vachalathiti, R. Smith. Patterns of spinal motion during walking. Gait Posture, 5 (1997), pp. 6-12

[19] M.P. Kadaba, H. Ramakrishnan, M. Wootten. Measurement of lower extremity kinematics during level walking. J Orthop Res, 8 (1990), pp. 383-392

[20] C. Mazza, M. Iosa, P. Picerno, A. Cappozzo. Gender differences in the control of the upper body accelerations during level walking. Gait Posture, 29 (2009), pp. 300-303

[21] C. Mazza, M. Zok, A. Cappozzo. Head stabilization in children of both genders during level walking. Gait Posture, 31 (2010), pp. 429-432

[22] J. Roislien, O. Skare, M. Gustavsen, N.L. Broch, L. Rennie, A. Opheim. Simultaneous estimation of effects of gender, age and walking speed on kinematic gait data. Gait Posture, 30 (2009), pp. 441-445

[23] K.A. Boyer, G.S. Beaupre, T.P. Andriacchi. Gender differences exist in the hip joint moments of healthy older walkers. J Biomech, 41 (2008), pp. 3360-3365

[24] W.J. Hurd, T.L. Chmielewski, M.J. Axe, I. Davis, L. Snyder-Mackler. Differences in normal and perturbed walking kinematics between male and female athletes. Clin Biomech (Bristol, Avon), 19 (2004), pp. 465-472

[25] K.A. McKean, S.C. Landry, C.L. Hubley-Kozey, M.J. Dunbar, W.D. Stanish, K.J. Deluzio. Gender differences exist in osteoarthritic gait. Clin Biomech, 22 (2007), pp. 400-409

[26] C.C. Prodromos, Y. Han, J. Rogowski, B. Joyce, K. Shi. A meta-analysis of the incidence of anterior cruciate ligament tears as a function of gender, sport, and a knee injury-reduction regimen. Arthroscopy, 23 (2007), pp. 1320-1325.e6 (The Journal of Arthroscopic \& Related Surgery)

[27] R.B. Fillingim, C.D. King, M.C. Ribeiro-Dasilva, B. Rahim-Williams, J.L. Riley III. Sex, gender, and pain: a review of recent clinical and experimental findings. J Pain, 10 (2009), pp. 447-485

[28] K.L. Johnson, L.G. Tassinary. Perceiving sex directly and indirectly: meaning in motion and morphology. Psychol Sci, 16 (2005), pp. 890-897

[29] C.-Y. Chang, T.-H. Wu. Using gait information for gender recognition. Intelligent Systems Design and Applications (ISDA), 2010. 10th International Conference on: IEEE (2010), pp. 1388-1393

[30] Y.S. Wong, C.W. Tam, S.M. Lee, C.P. Chan, H. Fu. Video-base people counting and gender recognition. Advances in swarm intelligence, Springer Berlin, Heidelberg (2012), pp. 228-235 
[31] R. McDonnell, S. Jörg, J.K. Hodgins, F. Newell, C. O'Sullivan. Virtual shapers \& movers: form and motion affect sex perception. Proceedings of the fourth symposium on Applied perception in graphics and visualization: ACM (2007), pp. 7-10

[32] R.B. Davis III, S. Ounpuu, D. Tyburski, J.R. Gage. A gait analysis data collection and reduction technique. Hum Mov Sci, 10 (1991), pp. 575-587

[33] G. Rab, K. Petuskey, A. Bagley. A method for determination of upper extremity kinematics. Gait Posture, 15 (2002), pp. 113-119

[34] M. Harrington, A. Zavatsky, S. Lawson, Z. Yuan, T. Theologis. Prediction of the hip joint centre in adults, children, and patients with cerebral palsy based on magnetic resonance imaging. J Biomech, 40 (2007), pp. 595-602

[35] A.L. Hof. Scaling gait data to body size. Gait Posture, 4 (1996), pp. 222-223

[36] J. Kuhtz-Buschbeck, K. Brockmann, R. Gilster, A. Koch, H. Stolze. Asymmetry of armswing not related to handedness. Gait Posture, 27 (2008), pp. 447-454

[37] M.S. Orendurff, A.D. Segal, G.K. Klute, J.S. Berge, E.S. Rohr, N.J. Kadel. The effect of walking speed on center of mass displacement. J Rehabil Res Dev, 41 (6A) (2004), pp. 829-834

[38] D.J. Lick, K.L. Johnson, S.V. Gill. Deliberate changes to gendered body motion influence basic social perceptions. Soc Cognit, 31 (2013), pp. 656-671

[39] M.H. Schwartz, A. Rozumalski, J.P. Trost. The effect of walking speed on the gait of typically developing children. J Biomech, 41 (2008), pp. 1639-1650

[40] D.M. Fessler, K.J. Haley, R.D. Lal. Sexual dimorphism in foot length proportionate to stature. Ann Hum Biol, 32 (2005), pp. 44-59

[41] P. Meyns, S.M. Bruijn, J. Duysens. The how and why of arm swing during human walking. Gait Posture, 38 (2013), pp. 555-562

[42] R.J. Kuczmarski, M.D. Carroll, K.M. Flegal, R.P. Troiano. Varying body mass index cutoff points to describe overweight prevalence among US adults: NHANES III (1988 to 1994). Obesity Res, 5 (1997), pp. 542-548

[43] R.W. Bohannon, A. Williams Andrews. Normal walking speed: a descriptive meta-analysis. Physiotherapy, 97 (2011), pp. 182-189

[44] M.A. Hunt, T.V. Wrigley, R.S. Hinman, K.L. Bennell. Individuals with severe knee osteoarthritis (OA) exhibit altered proximal walking mechanics compared with individuals with less severe OA and those without knee pain. Arthritis Care Res, 62 (2010), pp. 1426-1432 
[45] A. Mündermann, J.L. Asay, L. Mündermann, T.P. Andriacchi. Implications of increased medio-lateral trunk sway for ambulatory mechanics. J Biomech, 41 (2008), pp. 165-170

[46] H. Chiba, S. Ebihara, N. Tomita, H. Sasaki, J.P. Butler. Differential gait kinematics between fallers and non-fallers in community-dwelling elderly people. Geriatr Gerontol Int, 5 (2005), pp. $127-134$ 